
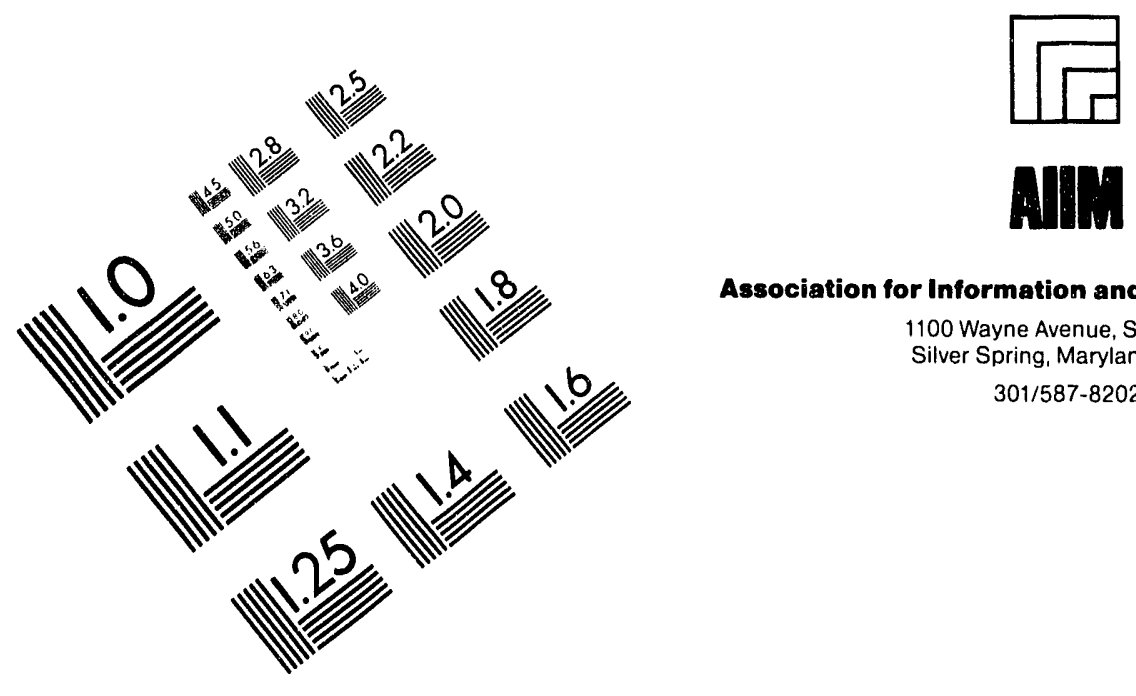

Association for Information and Image Management

1100 Wayne Avenue, Suite 1100

Silver Spring, Maryland 20910

301/587-8202

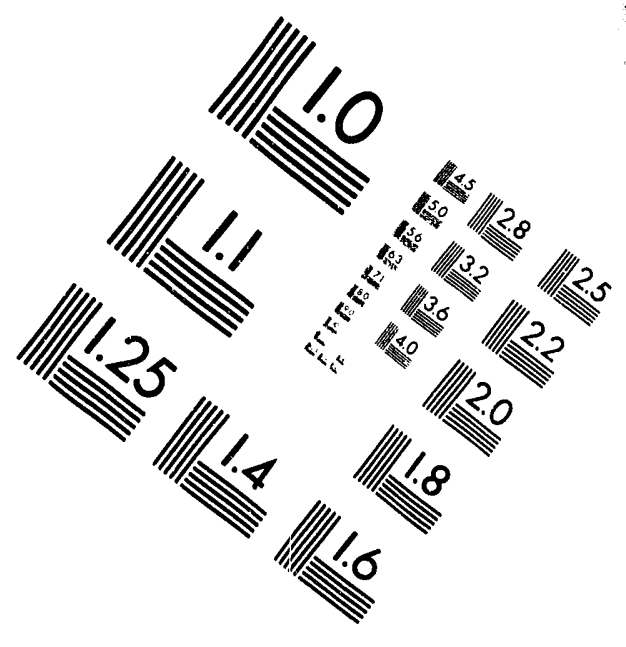

Centimeter

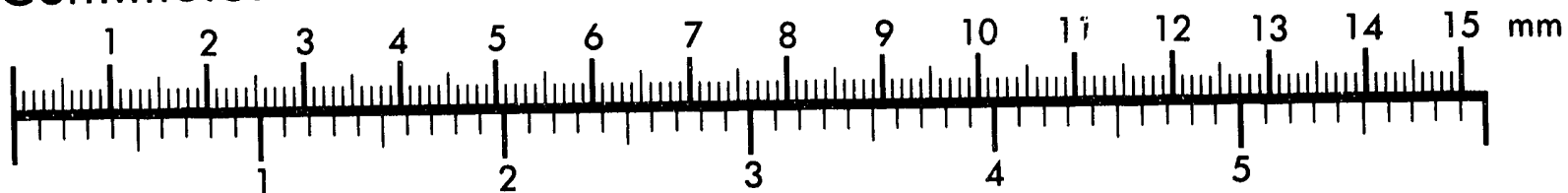

Inches
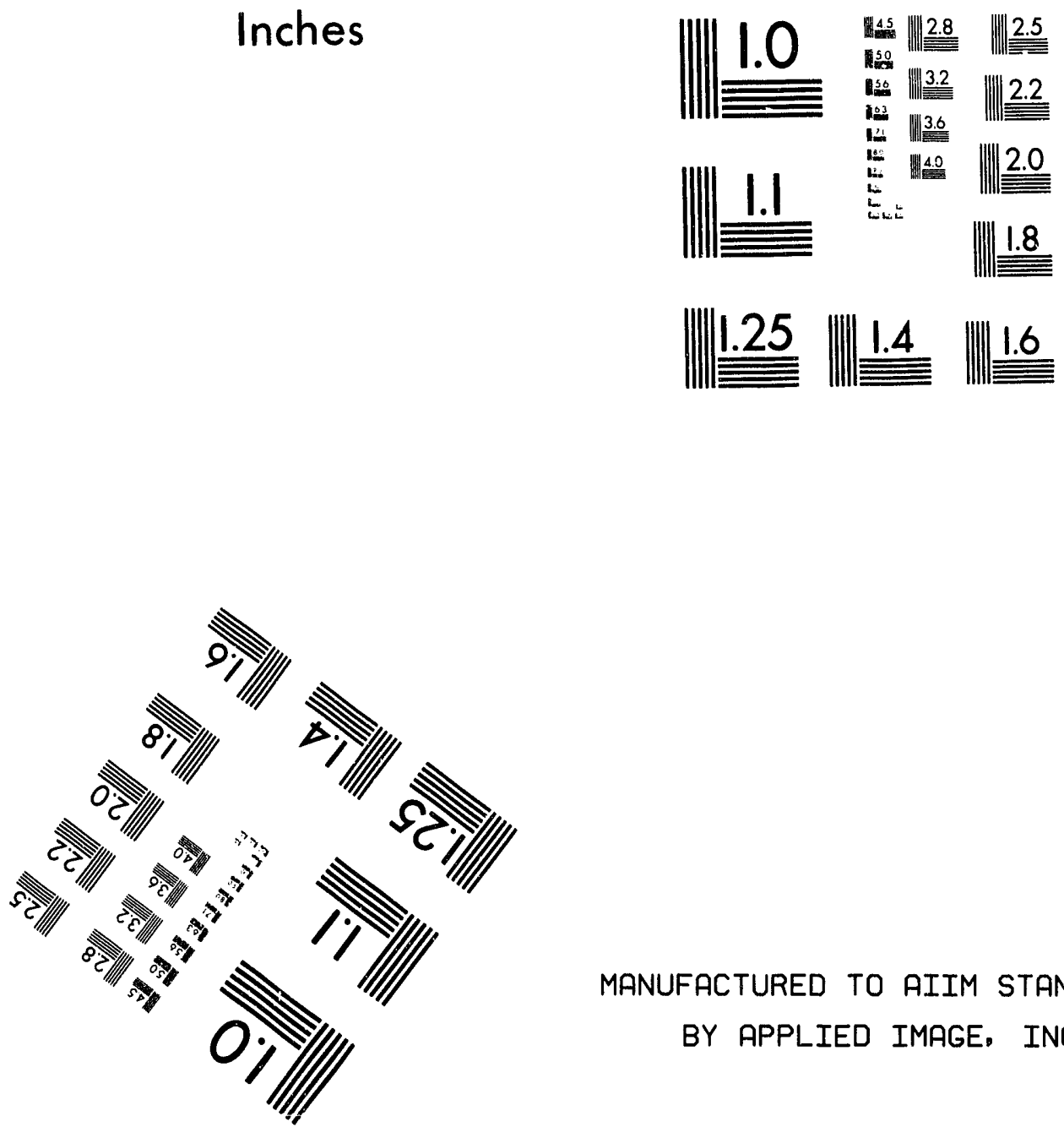

MANUFACTURED TO AIIM STANDARDS

BY APPLIED IMAGE, INC.

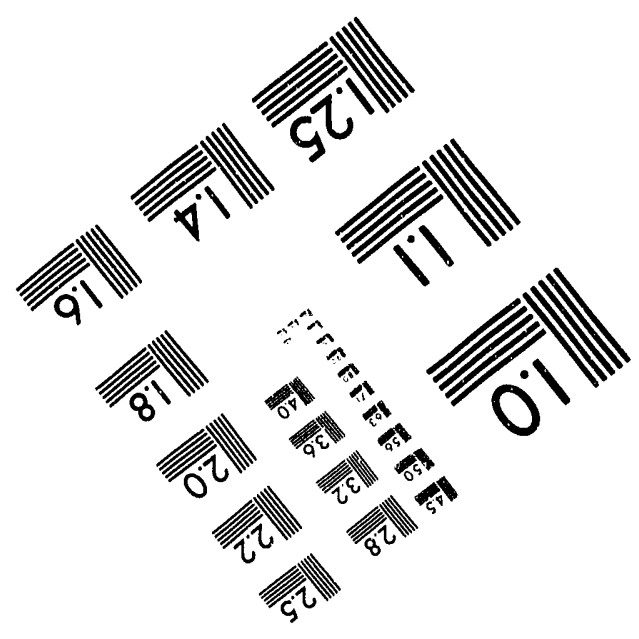



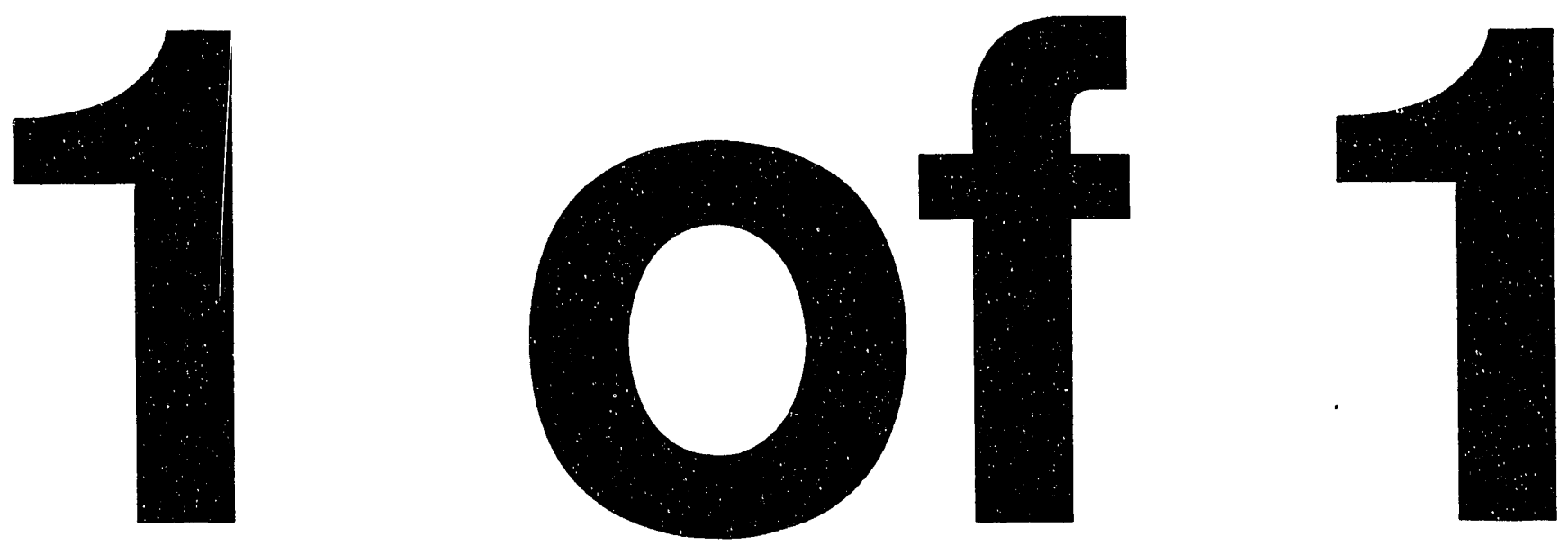


\title{
Decoupling Schemes for the SSC Collider ${ }^{*}$
}

\author{
Y. Cai, G. Bourianoff, B. Cole, R. Meinke, J. Peterson, F. Pilat, \\ S. Stampke, M. Syphers and R. Talman
}

Superconducting Super Collider Laboratory ${ }^{\dagger}$

2550 Beckleymeade Ave.

Dallas, TX 75237

May 1993

\section{MASTER}

*To be presented at the 1993 IEEE Particle Accelerator Conference on May 17-20, Washington, D.C.

†Operated by the Universities Research Association, Inc., for the U.S. Department of Energy under Contract No. DE-AC35-89ER40486. 


\title{
Decoupling Schemes for the SSC Collider
}

\author{
Y. Cai, G.Bourianoff, B.Cole, R.Meinke, J. Peterson, F. Pilat, S. Stampke, M.Syphers and R.Talman \\ SSC Laboratory, 2550 Beckleymeade Avenue, Dallas, Texas 75237 USA*
}

\section{Abstract}

A decoupling system is designed for the SSC Collider. This system can accommodate three decoupling schemes by using 44 skew quadrupoles in the different configurations. Several decoupling schemes are studied and compared in this paper.

\section{INTRODUCTION}

The SSC Collider consists of two arcs and two clusters and has two-fold symmetry. There are three insertions, two interaction regions and one utility region in each cluster. Two approved experiments, SDC and GEM, are located in the east cluster. Injection lines from the high energy booster, the beam abort system and RF system reside in the west utility. Other than some small bending regions between the insertions to deflect neutral particles from experiments, most bending is accomplished in arc. There are 196 FODO (90 degree) cells in each arc.

In an ideal synchrotron accelerator containing dipoles, quadrupoles and sextupoles with no alignment errors or multipole errors, motion of a particle in the horizontal plane is decoupled from its motion in vertical plane. This fact makes an ideal accelerator very simple to understand, therefore, easy to operate. In a real accelerator, the linear motions are always coupled due to skew quadrupole $\left(a_{1}\right)$ errors. In the case of the SSC Collider, there are three major sources of skew quadrupole errors. Main superconducting dipole magnets produce a skew quadrupole component when up-down symmetry is broken during a manufacturing process. Even with perfect symmetry, there are still sextupole $\left(b_{2}\right)$ errors due to persistence current in superconductors. These sextupole errors will feed down to skew quadrupole if particles do not pass the center of the dipole vertically. The second source is roll of main quadrupole magnets when aligned or fabricated. The last one is due to

Table 1

Sources of Linear Coupling

\begin{tabular}{|l|l|l|l|}
\hline \multirow{2}{*}{$\mathrm{a}_{1}$ in dipole } & sys. & $0.10 \mathrm{unit}$ & $0.5 \mathrm{~T}$ \\
\cline { 2 - 4 } & $\mathrm{rms}$. & $1.25 \mathrm{unit}$ & $2.8 \mathrm{~T}$ \\
\hline roll of quads & $\mathrm{rms}$. & $0.5 \mathrm{mrad}$ & $1.1 \mathrm{~T}$ \\
\hline $\begin{array}{l}\text { Vertical disp... } \\
\text { sexts. }\end{array}$ & $\mathrm{rms} \_c o r$ & $0.33 \mathrm{~mm}$ & $1.2 \mathrm{~T}$ \\
\cline { 2 - 4 } & rms_b $_{2}$ & $1.00 \mathrm{~mm}$ & $0.9 \mathrm{~T}$ \\
\hline
\end{tabular}

* Operated by the Universities Research Association, Inc., for the U. S. Department of Energy under Contract No. DE-AC3589ER40486 sextupole corrector magnets when misaligned vertically. The total skew quadrupole errors in a half cell at collision energy are tabulated in the last column (GL in Tesla) of Table 1.

Table 1 shows that dipole magnets are the dominant sources of linear coupling simply because of the number of dipole magnets in the Collider. Those errors are used in simulations in later sections.

\section{DECOUPLING SCHEMES}

In a coupled circular accelerator, fractional tunes of horizontal and vertical betatron motions can not be operated closely. Two families of skew quadrupoles are needed to reduce the band width (difference resonance) between fractional tunes so that fractional tunes can be operated closely to avoid other resonances. For this reason, almost all circular accelerators use this scheme to decouple vertical and horizontal motions. Operationally, this scheme is also very simple since changing and measuring tunes required in this scheme are routine operations. We will adopt it as a basic operation in the decoupling system because it is a proven scheme.

A two family decoupling scheme only reduces the width of difference resonance caused by skew quadrupole errors. It is known that skew quadrupole errors also contribute to a sum resonance. For a strongly coupled machine, the linear sum resonance can also cause some secondary effects, for example distorted beta function and residual tune split. To correct the secondary effects, an additional two families of skew quadrupoles are needed. Operationally, sum resonances can be minimized in the same way as the difference resonance. However, there is another way to use four families of skew quadrupoles by minimizing four independent coupling matrices elements in the one-turn matrix at a selected location. In this scheme two kickers with difference of $90^{\circ}$ in betatron phase are needed in each plane to measure the coupling matrix. It can be shown that these two methods of using four families are nearly equivalent. The second one is used in our simulations.

Transverse linear betatron motion can be described by a four by four symplectic matrix. This matrix always can be block diagonalized into two by two matrices[1][2]. The motion described by the diagonalized matrix is called eigen motion. The planes defined by the eigen motion with a small amplitude are called the eigen planes. The angles between the eigen planes and the horizontal or vertical planes can be measured by analyzing the reading of beam position monitors (BPMs) while the beam is excited horizontally or vertically. Therefore, the eigen angles at a beam position monitor location can be minimized by adjusting nearby skew quadrupole magnets accordingly [3]. This scheme requires that every skew quadrupole magnet have its own power supply and be controlled independently. 


\section{DECOUPLING SYSTEM}

The SSC collider is very different from any other accelerators because its size. In particular, it has very long arcs. Coupling can be built up gradually in the 196 cells to intolerable levels. For instance, to the point where eigen angle approaches $45^{\circ}$ so that there is no way to distinguish the horizontal motion from the vertical. Due to this fact, some skew quadrupoles in arcs are needed to reduce the build up of coupling. In the Collider decoupling system, there are five evenly distributed pairs of skew quadrupole magnets at missing dipole locations [4] in each arc. They will be used to minimize eigen angles of BPMs locations in the arcs.

Betatron tune adjustment will be made by two families of trim quadrupoles in the arcs. Therefore, differences in phase among skew quadrupoles in arcs will not be kept in constant when the Collider is tuned. As a result, skew quadrupoles in the arcs cannot be grouped into families because the final operating tunes for the Collider will be determined only after its commissioning. The correction systems have to accommodate a range of \pm 3 unit in tunes.

On the other hand, phase advances in each cluster will be fixed as the Collider is tuned. This is crucial for many interaction region correction schemes. For example, non-linear chromatic effects from triplets near an interaction point can be corrected by sextupoles at ends of arcs. To take advantage of this fact, four skew quadrupole magnets are placed inside or near each insertion in the clusters so that they can be grouped into four families based on their phases. Two families of skew quadrupoles for the difference resonance correction are at the middle of an empty half cell to maximize the difference of phases between horizontal and vertical betatron oscillations. The skew quadrupole magnets near interaction regions are located outside the tuning quadrupole magnets so that the phase relationship among skew quadrupoles will not be changed during a beta squeeze. This makes the decoupling sequence orthogonal to the beta squeeze sequence during the Collider operation.

These families are formed in control software, and can be rearranged in different clusters if the tunes of the Collider are changed.

\section{RESULTS}

The different decoupling schemes described above have been simulated by using the tracking code TEAPOT on the same lattice and with the same set of alignment errors and multipole errors so that the comparison can be made directly. Betatron tunes in simulations are $v_{1}=123.785$, and $v_{1}=122.765$. The selection of the difference of one unit in integer part of tunes is to suppress the effects from systematic $a_{1}$ in main dipole magnets. Because the Collider has two fold symmetry, systematic errors only contribute to even integer resonances. A summary of simulation results is tabulated in Table2.

Table 2 shows that 24 skew quadrupoles in four families are adequate to minimize tune separation. However, controlling eigen angles within \pm 10 degree is also very important for simple operation of the Collider. The next four figures show that only one scheme can achieve the control of eigen angles with 44 individually set skew quadrupoles by using the eigen angle minimization scheme. Schemes based on families only can control eigen angles in a very limited way. Eigen angles accunulate in arcs and exceed $10^{\circ}$ when no skew quadrupole magnets are in the arcs.
Table 2

Minimum Tune Separation

\begin{tabular}{|l|c|c|}
\hline $\begin{array}{c}\text { Decoupling } \\
\text { Schemes }\end{array}$ & $\begin{array}{c}\text { Number of } \\
\text { skew quads }\end{array}$ & $\begin{array}{c}\text { Minimum } \\
\text { tune } \\
\text { separation }\end{array}$ \\
\hline No correction & 0 & 0.024 \\
\hline Two families & 12 & 0.010 \\
\hline Four families & 24 & 0.003 \\
\hline $\begin{array}{l}\text { Minimizing } \\
\text { eigen angles }\end{array}$ & 44 & 0.0005 \\
\hline
\end{tabular}

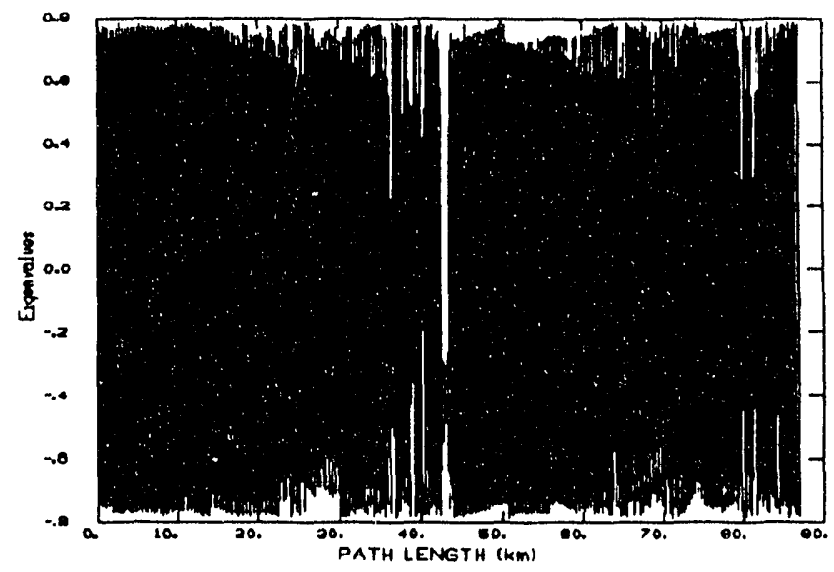

Figure 1. Eigen angles in radian with no coupling correction.It shows that eigen angles reach 45 degree at many locations in the Collider and the motions in different planes are coupled very strongly.

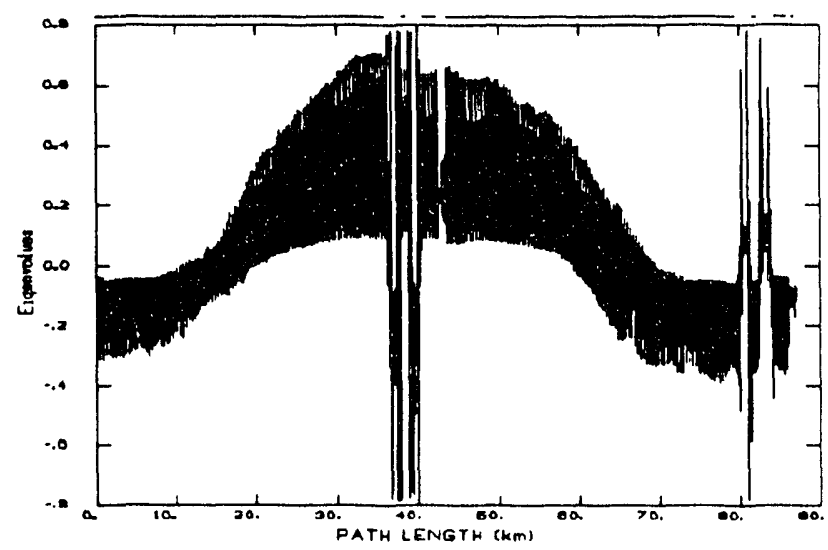

Figure 2. Eigen angles in radians with two families of 12 skew quadrupoles in clusters. It shows that eigen angles have been brought under control. However, eigen angles accumulate in arcs and reach $45^{\circ}$ at the end of arcs. 

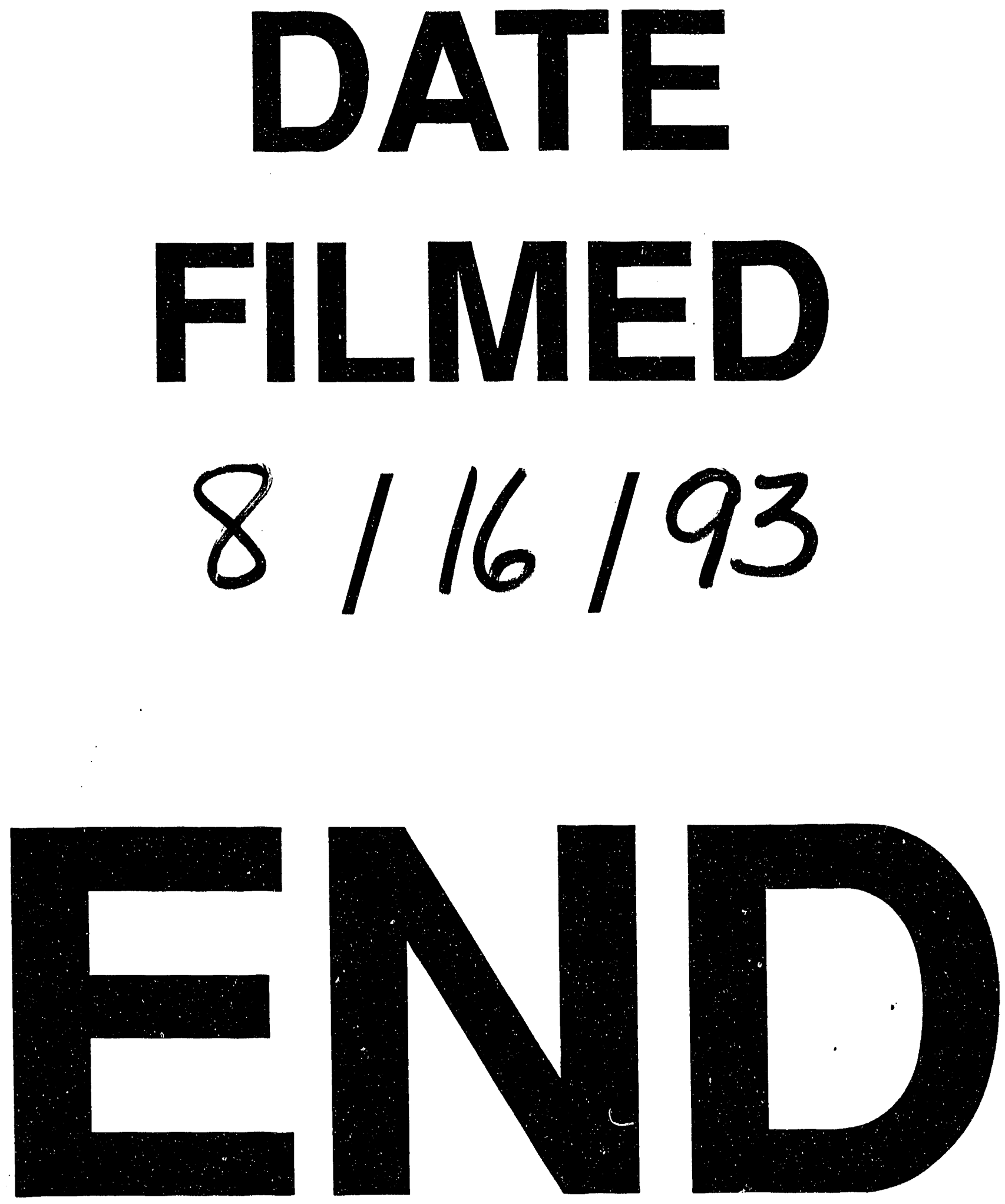
